\title{
Freezing Transition in Very Small Systems of Hard Spheres
}

\author{
Willem K. Kegel, ${ }^{1}$ Howard Reiss, ${ }^{2}$ and Henk N. W. Lekkerkerker ${ }^{1}$ \\ ${ }^{1}$ Van't Hoff Laboratory for Physical and Colloid Chemistry, Debye Institute, Utrecht University, Padualaan 8 , \\ 3584 CH Utrecht, The Netherlands \\ ${ }^{2}$ Department of Chemistry and Biochemistry, University of California at Los Angeles, Los Angeles, California 90095
}

(Received 4 June 1999)

\begin{abstract}
By applying a rigorous formalism, the grand distribution function of very small systems of hard spheres is obtained. The hard sphere freezing transition appears as two peaks of this function. The formalism requires input of the available volume, i.e., the configurationally averaged volume of a system that is available for an additional sphere center. These volumes are computed numerically. We show that by this treatment the freezing transition (1) follows "naturally," i.e., properties of the fluid and the solid phases need not be inserted into the treatment in advance; (2) is already apparent in systems containing a number of spheres as small as eight; and (3) is caused by the system avoiding configurations that can best be characterized as "defective solids."
\end{abstract}

PACS numbers: 64.60.Cn, 64.70.Dv

A considerable fraction of the elements, when compressed, undergo a first order phase transition from a disordered state (a gas, fluid, or liquid) to a face-centered cubic (fcc) or hexagonal close packed (hcp) crystal. Computer simulations [1-3], as well as experiments [4] show that this freezing transition is already apparent in the simplest model of interacting atoms: a collection of hard spheres subject to thermal agitation, implying that crystallization requires only excluded volume interactions. To date, there is no rigorous statistical mechanical proof of the hard sphere freezing transition, and even simulation is uncertain in the coexistence region. Theory can explain only the transition "afterwards"; that is, properties of the coexisting phases should be inserted into the theories in advance, and the coexisting densities follow by minimizing a free energy functional, or, equivalently, requiring the chemical potentials and the pressures of the two branches to be equal in the coexistence region. These theories are often quite accurate quantitatively; i.e., the coexisting densities and the pressure of the coexisting region are in good agreement with computer simulations; see, for example, [5]. However, from a fundamental viewpoint they are still unsatisfactory. The splitting of a hard sphere system into a fluid and a solid branch at a certain density is, evidently, hidden in the partition function of the system. A thorough theory of hard sphere freezing should therefore identify this property of the partition function that leads to the observed symmetry breaking.

In this work we take a new approach to the freezing transition and apply it to very small systems of hard spheres. On the one hand, treating small systems may be considered as a "step back" compared to theories and simulations that concentrate on the thermodynamic limit. The reward, however, is that we show that in small systems, a complete treatment is possible, and thermodynamic properties within the coexistence region can be obtained. To this end, we concentrate on the available volumes of these systems. We compute these volumes numerically as a func- tion of the number of spheres, at several constant system volumes. It is shown that at a certain point, the available volume increases with the number of spheres. Making use of an exact relation between available volume and the partition function, this behavior is shown to correspond to a first order phase transition in the grand ensemble. Interestingly, this behavior is already apparent in extremely small systems.

Rigorous proof of a first order phase transition (i.e., a singularity in the appropriate thermodynamic functions) requires knowledge of the grand partition function [6]

$$
\Xi=\sum_{N=0}^{N_{\max }} z^{N} q_{N}
$$

In this equation, the reduced activity $z=\left(D^{3} / \Lambda^{3}\right) e^{\beta \mu}$, where $\mu$ is the chemical potential, $\beta=1 / k T$, and $k$ and $T$ are Boltzmann's constant and the absolute temperature, respectively. The quantities $\Lambda$ and $D$ are the thermal de Broglie wavelength of a hard sphere and its diameter. It can be shown [7] that the (reduced) canonical partition function of a system containing $N$ hard spheres is exactly given by the recursive relation

$$
q_{N}=\frac{v_{0}^{(N-1)}}{N} q_{N-1}
$$

where $v_{0}^{(N)}=V_{0}^{(N)} / D^{3}, V_{0}^{(N)}$ denoting the available volume of the system. This quantity is the configurationally averaged volume that is available for the center of an $(N+$ 1)th hard space in a system containing $N$ hard spheres in a volume $V$, and has a purely geometric character. Indeed, the idea that the thermodynamic properties of hard sphere systems is a problem of statistics, dominated by the constraints of geometry, forms the basis of "scaled particle theory" and "statistical geometry"; see [8,9], and references therein. We note that $v_{0}^{(N)}$ is different from the "free volume" (see [10], and references therein) which is defined 
as the space available to a particle with its neighbors held fixed.

A singularity in the thermodynamic functions can appear only when $V \rightarrow \infty$ [6]. In this thermodynamic limit, the summation in Eq. (1) runs up to $N_{\max } \rightarrow \infty$. However, in this work we are concerned with only systems where $V$, and thus $N_{\max }$, is finite, the last mentioned value being the maximum number of spheres that can be crammed into $V$. In finite systems, a steep change of the thermodynamic functions, rather than a singularity, suggests a first order phase transition [11]. Thus, a first order phase transition can be apparent from a steep change in the average number of spheres in a box,

$\langle N\rangle=\frac{\sum_{N=0}^{N_{\max }} N z^{N} q_{N}}{\Xi}=\sum_{N=0}^{N_{\max }} N P_{N}=\left(\frac{\partial \ln \Xi}{\partial \ln (z)}\right)_{T V}$,

as a function of the activity. This equation defines the probability that a box contains $N$ spheres, referred to as the grand distribution function,

$$
P_{N}=\frac{z^{N} q_{N}}{\Xi}
$$

Two peaks of the grand distribution function, at some value of the activity, are very strong evidence of a first order phase transition in small systems [12]. We note that it is prohibitively difficult, in dense systems, to evaluate the grand distribution function directly by computer simulation (using the Gibbs ensemble).

We computed the available volumes of $N$ hard spheres in a box with a fixed geometry and different (fixed) volumes, using a Monte Carlo procedure. In principle, however, this quantity may be calculated analytically. The box envelops the centers of the spheres, so that part of a sphere may stick out of the box. We choose $N_{\max }=8$ and $N_{\max }=27$. It is convenient to express the volume of the boxes relative to the volume of their close packed geometry, by defining $\alpha=v_{0}^{(0)} /(1 / \sqrt{2})$ if $N_{\max }=8$, and $\alpha=$ $v_{0}^{(0)} / 4 \sqrt{2}$ if $N_{\max }=27$, where the values of the denominators reflect the reduced volume of the boxes (with the proper geometry) in which $N_{\max }$ spheres are close packed. For the system with $N_{\max }=8, \alpha=1$ corresponds to the smallest possible close packed crystal (fcc or hcp). Of course, many other geometries of the boxes are possible, and a systematic study will be published later, as indeed, (very) small systems show interesting behavior in itself; see, for example, [13]. However, no qualitatively different behavior was observed in other geometries. We avoid forcing the systems to order into a crystal at $N=N_{\max }$ by setting $\alpha \geq 1.3$ for both sizes. The available volumes are plotted in Fig. 1.

It can be seen from Fig. 1 that at the largest values of $\alpha$ that were used, the available volumes steadily decrease with $N$, as expected. At intermediate values of $\alpha$, the available volumes remain roughly constant with increasing $N$ for $N \geq 4\left(N_{\max }=8\right)$ and $N \geq 21\left(N_{\max }=27\right)$. At these values of $\alpha$, the value of $N_{\max }$ should be considered

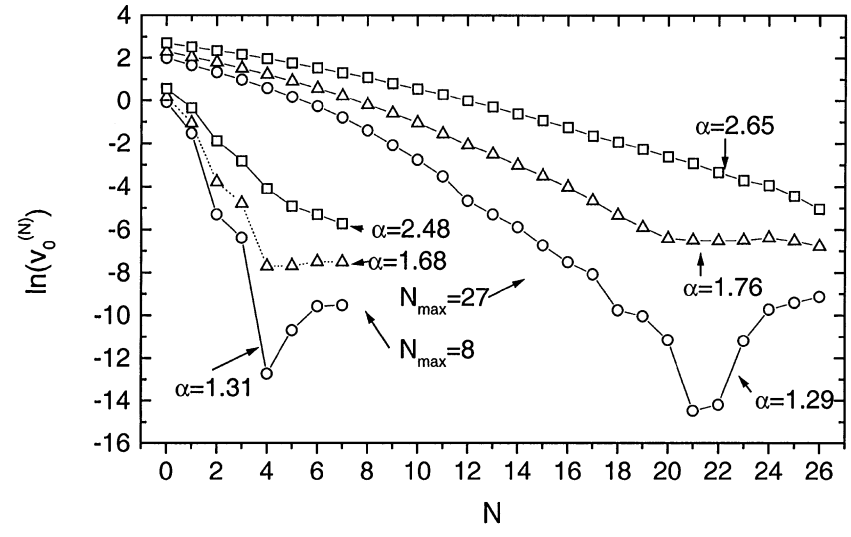

FIG. 1. The available volume as a function of the number of hard spheres in the system. System with $N_{\max }=8, \alpha=2.48$ (squares), $\alpha=1.68$ (triangles), and $\alpha=1.31$ (circles); also for the system with $N_{\max }=27$, and comparable values of $\alpha$ : 2.65 (squares), $\alpha=1.76$ (triangles), $\alpha=1.29$ (circles). Points are connected to guide the eye. Available volumes were averaged over at least $10^{4}$ configurations. For the smallest values of the available volumes, the uncertainty is of the same order of magnitude as the symbol size. For the other values the uncertainty is much smaller. Several different initial configurations were used, and no systematic variation of the values of the available volumes was observed, indicating that by this method representative fractions of configuration space were sampled.

as a constraint imposed on the system; i.e., the boxes can physically contain more than $N_{\max }$ spheres. This is not the case for the smallest values of $\alpha$ used here. At these values of $\alpha$, in the system with $N_{\max }=8$, when $N \geq 4$ obviously the available volume increases with $N$. The larger system, with $N_{\max }=27$ shows a similar trend. This, at first sight somewhat counterintuitive result, implies that the denser the system, the larger the insertion probability of an additional sphere. It will be shown later that this behavior corresponds to increasing order in the system. At this point we mention that it can be proved exactly by statistical geometrical methods [8], that if a first order transition occurs, the available volume must increase. On the other hand, such an increase in itself is not sufficient proof of a first order transition: this proof can follow only from the thermodynamic functions.

The relevant thermodynamic functions are shown in Figs. 2 and 3, where we plotted $\langle N\rangle$ versus $\ln (z)$, and the probability distributions at different $z$, respectively. It is obvious that for the smallest studied values of $\alpha$, the systems convincingly show the signature of the freezing transition, the transition being sharper (i.e., more like a singularity) for the larger system. The two peaks of the grand probability distribution function is the strongest evidence for this transition. It is rather surprising that even the smallest system $\left(N_{\max }=8\right)$ already shows the small-system analog of the freezing transition. This result emphasizes the geometric character of this transition. Comparison with the behavior of the available volume as a function of $N$, see Fig. 1, immediately reveals that the "jumps" of $\langle N\rangle$ in Fig. 2, as well as the values of $N$ 


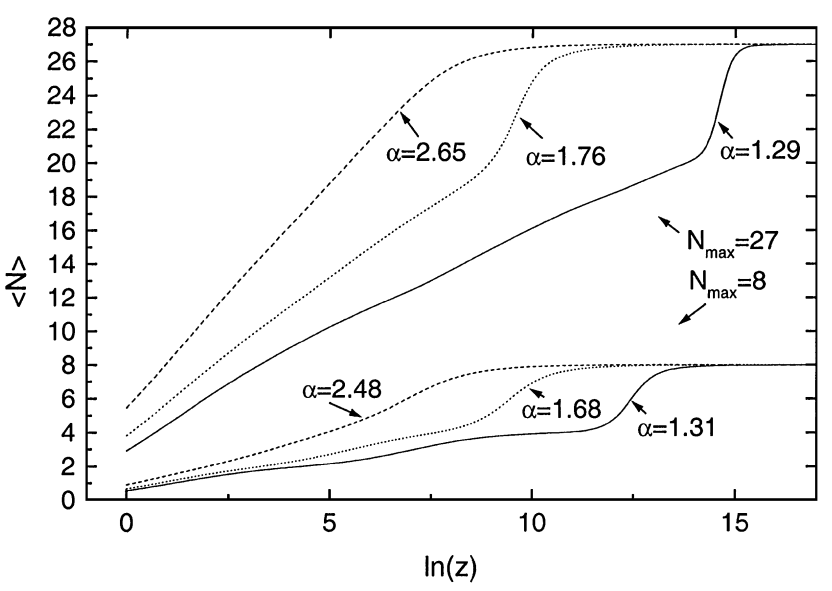

FIG. 2. Average number of spheres in a box, $\langle N\rangle$, as a function of the reduced activity, $\ln (z)$. System with $N_{\max }=8$, $\alpha=2.48$ (dashed line), $\alpha=1.68$ (dotted line), and $\alpha=$ 1.31 (solid line); also for the system with $N_{\max }=27$, and comparable values of $\alpha: 2.65$ (dashed line), $\alpha=1.76$ (dotted line), $\alpha=1.29$ (solid line). The steep increase of $\langle N\rangle$ points to the small-system analog of a first order phase transition.

in between the two peaks of the probability distribution, Fig. 3, correspond to the interval in $N$ where the available volume increases. Thus, the freezing transition appears as a consequence of the available volumes passing through a minimum as a function of the number of spheres. Before discussing this observation in terms of the structure of the system, we make a comparison to systems in the thermodynamic limit. We define the volume fraction of hard spheres in the system as $\phi=\left(N / N_{\max }\right) \alpha^{-1}(\pi / 3 \sqrt{2})$, where the last term on the right-hand side refers to the volume fraction where hard spheres are close packed in an fcc or hep structure. A proof showing that this value indeed reflects the most dense packing of spheres in three dimensions has recently been presented [14]; see also [15]. In the small systems studied here, however, volume fraction does not have the same significance as it has in the thermodynamic limit, where both $N$ and $V$ go to infinity at the same time, keeping their ratio constant. In the small system (with $N_{\max }=8$ ), taking the somewhat arbitrary smallest value of $\alpha$ used here, the values of the coexisting volume fractions are $\phi_{f}=$ 0.28 and $\phi_{m}=0.56$ (the subscripts denote the "freezing" and the "melting" volume fraction, respectively), while in the larger system (with $N_{\max }=27$, and again for the smallest value of $\alpha$ ), these volume fractions are $\phi_{f}=0.43$ and $\phi_{m}=0.57$. In the smallest system, in particular, the freezing volume fraction is far away from the values estimated for infinitely large systems [3], where $\phi_{f}=$ 0.494 and $\phi_{m}=0.545$. Clearly the freezing volume fraction in the system with $N_{\max }=27$ is much closer to the value for a "bulk" system. Computer simulations [16] indeed indicate that the freezing transition shifts to lower densities upon decreasing the system size. In the systems studied here, we speculate that there may be an additional effect due to the presence of the walls, where
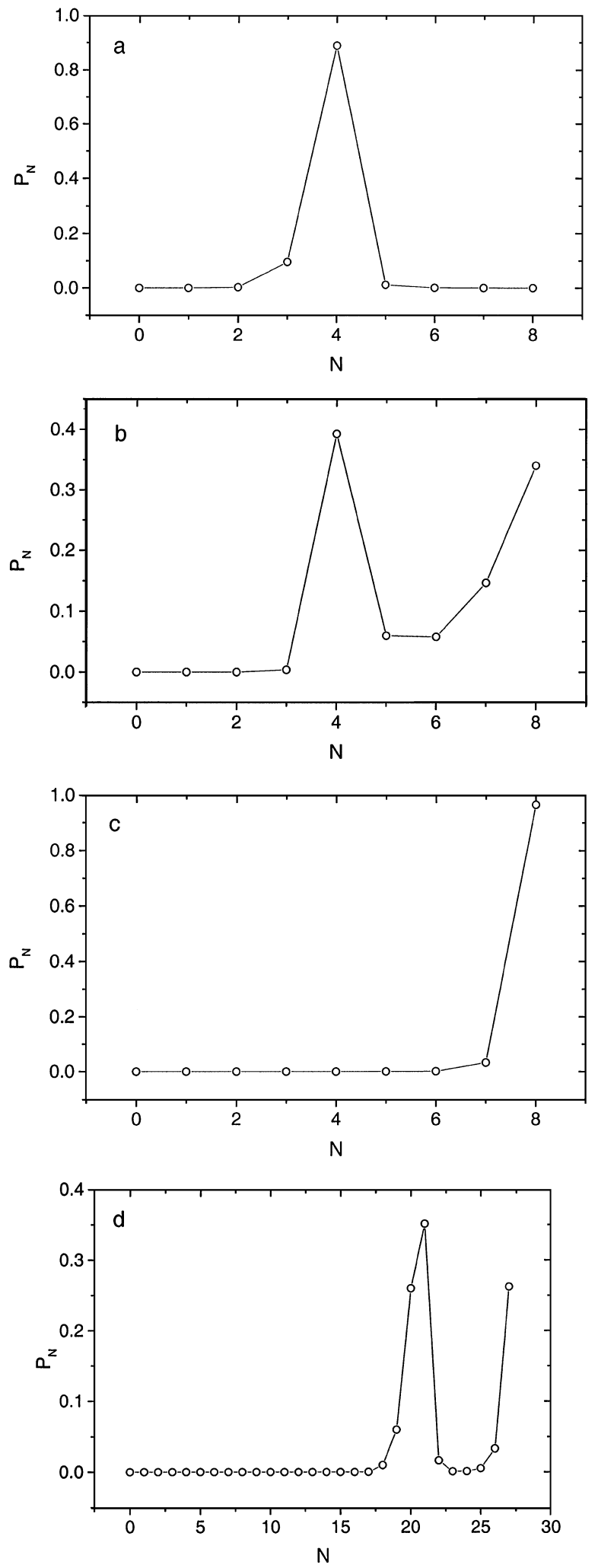

FIG. 3. Grand probability distribution $P_{N}$ vs $N$ for different values of $z$. System with $N_{\max }=8 ; \alpha=1.31$. (a) $\ln (z)=$ 10 ; (b) $\ln (z)=12.45$, corresponding to the maximum in $\sigma_{N}^{2}=$ $\left\langle N^{2}\right\rangle-\langle N\rangle^{2}$; (c) $\ln (z)=15$. (d) As (a)-(c), but now for the system with $N_{\max }=27 ; \alpha=1.29, \ln (z)=14.5$ (maximum in $\left.\sigma_{N}^{2}\right)$. Note that "intermediate" $P_{N}$ 's, in between the two peaks, become very close to 0 upon increasing the system size from $N_{\max }=8$ to 27 [compare (b)]. 

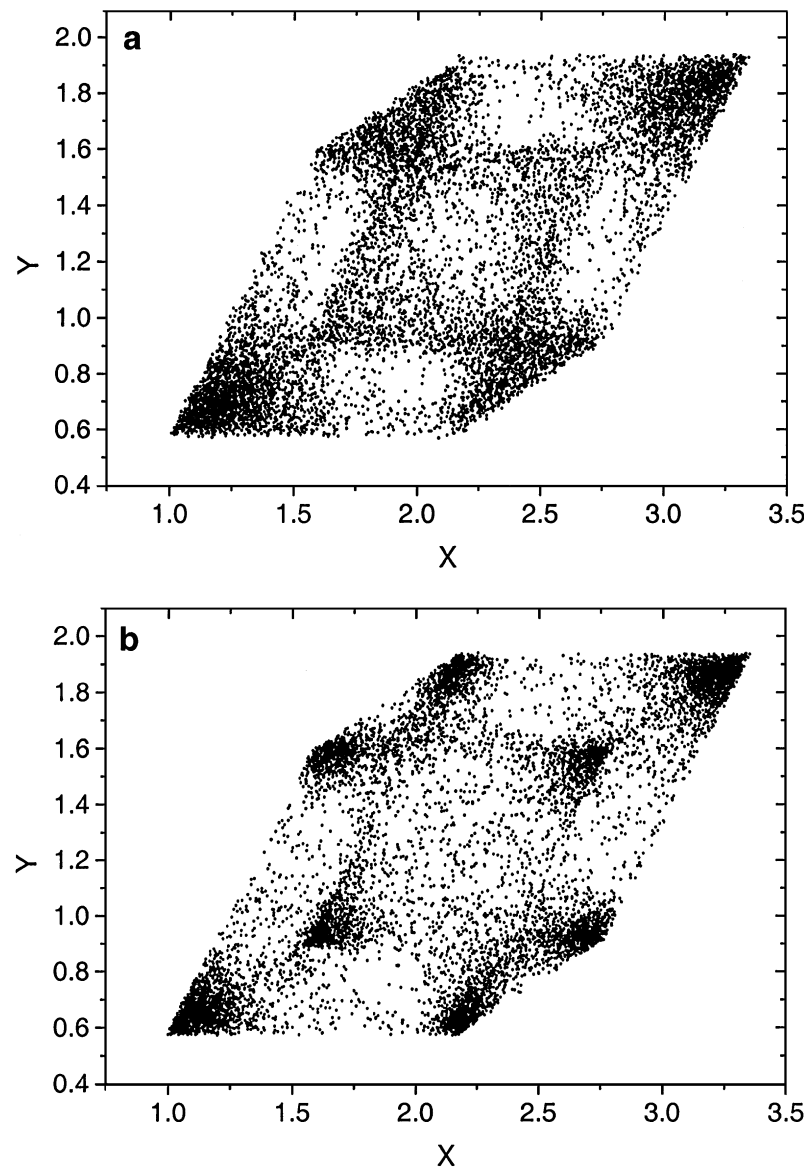

FIG. 4. Projection of the positions of sphere centers of $10^{4}$ configurations, in the plane where the layers are hexagonally packed at close packing. The coordinates are in units of the sphere diameter. System with $N_{\max }=8, \alpha=1.68, N=4$, corresponding to the minimum of $V_{0}^{(N)}$ (a), and $N=5$ (b).

hard spheres are observed to undergo "prefreezing"at a volume fraction smaller than the freezing volume fraction $[17,18]$. Both effects tend to broaden the coexistence region. We also compare the reduced activities, where the systems split into two densities, to the estimated value in the thermodynamic limit. The latter value [19] is $\ln (z)=$ 17.13, while the values found here $\operatorname{are} \ln (z)=12.45$ for the smaller system and $\ln (z)=14.5$ for the system with $N_{\max }=27$; see Fig. 3 (and again taking the smallest used value of $\alpha$ ). These values are not too far off and increase with system size.

From Figs. 2 and 3 it follows that the "dense branch" of the systems always corresponds to the highest possible density (i.e., $\langle N\rangle \approx N_{\max }$ ). We seek an explanation for this by plotting the positions of $N$ sphere centers of $10^{4}$ configurations in the system with $N_{\max }=8$, for $N=4$ and $N=5$, in Fig. 4, using $\alpha=1.68$ (where the first sign of the freezing transition appears). It is clear from Fig. 4 that in the system containing four spheres, the centers tend to occupy positions at the corners and along the walls, but there is no sign of ordering into distinct lattice positions. When $N=5$, on the other hand, the system preferentially occupies eight lattice positions and resembles a "defective solid" (there are only five spheres for eight lattice positions). A comparable behavior was observed in the system with $N_{\max }=27$. Thus, the increasing value of the available volume with $N$ (Fig. 1) is a result of "defects" that decrease in number but become more localized when $N$ increases. The defective solid configurations are clearly undesirable in the grand ensemble; i.e., their occupation numbers become very small; see Fig. 3. Note that these configurations were generated in the $(N V T)$ ensemble (i.e., the ensemble in which the available volume was evaluated). It is only at the point where the constraint of constant $N$ is replaced by a constant chemical potential that these (homogeneous) configurations become unstable.

The signature of the hard sphere freezing transition is already present in systems containing a number of spheres as small as eight. Further progress towards a noncomputational theory requires analytical expressions for the available volumes, a massive task ahead.

We are grateful to Dr. J.S. van Duijneveldt for performing preliminary computer simulations of a constrained system and to Dr. J. K. G. Dhont for critically reading the manuscript. The contribution of W. K. K. to the work presented here has been made possible by a fellowship of the Royal Netherlands Academy of Arts and Sciences.

[1] W. W. Wood and J. D. Jacobsen, J. Chem. Phys. 27, 1207 (1957).

[2] B. J. Alder and T. E. Wainwright, J. Chem. Phys. 27, 1208 (1957).

[3] W. G. Hoover and F.H. Ree, J. Chem. Phys. 49, 3609 (1968).

[4] P. N. Pusey and W. van Megen, Nature (London) 320, 340 (1986).

[5] M. Baus and J. F. Lutsko, Phys. A 176, 28 (1991).

[6] C. N. Yang and T. D. Lee, Phys. Rev. 87, 404 (1952).

[7] R. J. Speedy, J. Chem. Soc. Faraday Trans. 2 73, 714 (1977).

[8] H. Reiss and A. D. Hammerich, J. Phys. Chem. 90, 6252 (1986).

[9] H. Reiss, J. Phys. Chem. 96, 4736 (1992).

[10] W. G. Hoover, N.E. Hoover, and K. Hanson, J. Chem. Phys. 70, 1837 (1979).

[11] T.L. Hill, Thermodynamics of Small Systems, Part I (Dover, New York, 1994).

[12] T.L. Hill, Statistical Mechanics; Appendix 9 (McGrawHill, New York, 1956).

[13] Z. T. Németh and H. Löwen, J. Phys. Condens. Matter 10, 6189 (1998).

[14] T.C. Hales, http://www.math.Isa.umich.edu/ hales/ countdown.

[15] N. J. A. Sloane, Nature (London) 395, 435 (1998).

[16] R. J. Speedy, J. Phys. Condens. Matter 9, 8591 (1997).

[17] D. J. Courtemanche and F. van Swol, Phys. Rev. Lett. 69, 2078 (1992).

[18] D. J. Courtemanche, T. A. Pasmore, and F. van Swol, Mol. Phys. 80, 861 (1993).

[19] D. Frenkel and B. Smit, Understanding Molecular Simulation (Academic Press, San Diego, London, 1996). 